\title{
A new edited $k$-nearest neighbor rule in the pattern classification problem
}

\author{
Kazuo Hattori*, Masahito Takahashi \\ Department of Electrical Engineering and Electronics, Toyohashi University of Technology, Tempaku-cho, Toyohashi 441-8580, Japan
}

Received 29 October 1998; accepted 16 February 1999

\begin{abstract}
A new edited $k$-nearest neighbor $(k-\mathrm{NN})$ rule is proposed. For every sample $y$ in the edited reference set, all the $k$ - or $(k+\ell)$-nearest neighbors of $y$ must be in the class to which $y$ belongs. Here $\ell$ denotes the number of samples which tie with the $k$ th nearest neighbor of $y$ with respect to the distance from $y$. The performance of the rule proposed has been investigated using three classification examples. As a result, it is shown that the rule proposed will yield good results in many pattern classification problems. (C) 2000 Pattern Recognition Society. Published by Elsevier Science Ltd. All rights reserved.
\end{abstract}

Keywords: Pattern classification problems; $k$-Nearest neighbor rule; Wilson's edited $k$-nearest neighbor rule; Edited reference set; Number of samples misclassified

\section{Introduction}

In pattern classification problems, the $k$-nearest neighbor $(k-\mathrm{NN})$ rule [1-3] is a simple nonparametric decision rule. In the rule, an input sample is assigned to the class to which the majority among its $k$-nearest neighboring labeled samples are assigned. The $k-\mathrm{NN}$ rule $[1-3]$ has been widely used since it is effective, when probability distributions of the feature variables are not known, and therefore, Bayes decision rule [2] is not applicable.

Wilson [4] proposed an edited $k-\mathrm{NN}$ rule to improve the $1-\mathrm{NN}$ rule [1-3]. In his rule, editing the reference set is first performed: Each sample in the reference set is classified using the $k-\mathrm{NN}$ rule [1-3] and the set formed by eliminating it from the reference set. All the samples misclassified are then deleted from the reference set. Afterward, any input sample is classified using the 1-NN rule [1-3] and the edited reference set. Wilson's edited $k-\mathrm{NN}$ rule [4] has yielded good results in many finitesample-size problems, although its asymptotic optimality has been disproved $[5,6]$.

\footnotetext{
*Corresponding author. Fax: + 0532-44-6757
}

Dasarathy [7] has developed a condensing method to edit the reference set: His method provides the minimal consistent subset (MCS) which is used as the edited reference set. All the samples in the reference set can be correctly classified using the $1-\mathrm{NN}$ rule [1-3] and the MCS. Dasarathy [7] has shown that the performance of the $1-\mathrm{NN}$ rule [1-3] suffers little degradation when a given large reference set is replaced by its much smaller MCS. Kuncheva [5] has applied a genetic algorithm [8] to editing the reference set for the $k-\mathrm{NN}$ rule [1-3]. Some fitness functions for the genetic algorithm [8] have been proposed, and the fitness function including a penalizing term is found to be effective to edit the reference set.

In the editing method using the MCS [7] and that using the genetic algorithm $[5,8]$, the number of samples in the edited reference set is considerably small, and therefore, the computational burden to classify input samples is much reduced. However, classification accuracy in these two editing methods [5,7] is usually found not to be higher than that in the $k-\mathrm{NN}$ rule [1-3]. In Wilson's edited $k-\mathrm{NN}$ rule [4], the number of samples in the edited reference set is usually not so small. With respect to classification accuracy, however, his rule is usually better in practical classification problems than 
the editing method using the MCS [7] and that using the genetic algorithm $[5,8]$.

The purpose of this work is to propose a new edited $k-\mathrm{NN}$ rule. In the rule proposed, high classification accuracy is more weighted than decrease in the number of samples in the reference set. Three classification examples are presented to test the rule proposed. The performance of the rule proposed has been compared with those of the $k-\mathrm{NN}$ rule [1-3] and Wilson's edited $k-\mathrm{NN}$ rule [4].

\section{A new edited $k$-NN rule}

In this section, a new edited $k-\mathrm{NN}$ rule has been proposed. Let $W_{0}=\left\{x_{1}, x_{2}, \ldots, x_{n}\right\}$ be the set of $n$ labeled samples, namely, a reference set. In the rule proposed, the reference set $W_{0}$ is edited as follows:

E-1. For each labeled sample $x_{i}, 1 \leqslant i \leqslant n$, find the $k$ nearest neighbors of $x_{i}$ from the set $W\left(x_{i}\right)$ which is given by $\left\{x_{1}, x_{2}, \ldots, x_{i-1}, x_{i+1}, \ldots, x_{n}\right\}$. Let $d_{j}\left(x_{i}\right)$ be the distance from $x_{i}$ to its $j$ th nearest neighbor, and assume $d_{1}\left(x_{i}\right) \leqslant d_{2}\left(x_{i}\right) \leqslant \cdots \leqslant d_{k}\left(x_{i}\right)$. Let $V\left(k, x_{i}\right)$ be the set of the $k$-nearest neighbors of $x_{i}$. If $\ell$ different labeled samples in $W\left(x_{i}\right)$ satisfy the condition that the distance from $x_{i}$ to each of them is equal to $d_{k}\left(x_{i}\right)$, include these $\ell$ labeled samples in $V\left(k, x_{i}\right)$, i.e., obtain the $(k+\ell)$-nearest neighbors of $x_{i}$. Check the condition that all the samples in $V\left(k, x_{i}\right)$ should be assigned to the class to which $x_{i}$ is assigned. If the condition is satisfied, take $x_{i}$ as a typical sample in the class to which $x_{i}$ belongs; otherwise, $x_{i}$ is not any typical sample.

E-2. Obtain the set of all the typical samples as the edited reference set $W_{1}(k)$.

Any input sample is classified using the $k^{\prime}-\mathrm{NN}$ rule [1-3] and $W_{1}(k)$, where $k^{\prime}$ is not restricted to 1 . In the practical use of the rule proposed, the values of $k$ and $k^{\prime}$ should be optimally determined as follows:

$\mathrm{K}-1$. Determine the range of $k$ and that of $k^{\prime}$, properly. Express any possible pair of $k$ and $k^{\prime}$ as $\left(k, k^{\prime}\right)$.

K-2. For each pair $\left(k, k^{\prime}\right)$, obtain $W_{1}(k)$ and classify each sample $x$ in $W_{0}$ using the $k^{\prime}-\mathrm{NN}$ rule [1-3] and the set $W_{1}(k, x)$. Here $W_{1}(k, x)$ is the set formed by eliminating $x$ from $W_{1}(k)$, if $x$ is in $W_{1}(k)$. If $x$ is not in $W_{1}(k)$, the set $W_{1}(k, x)$ is given by $W_{1}(k)$. Evaluate the number of samples misclassified for each pair $\left(k, k^{\prime}\right)$.

K-3. Find the pair $\left(k_{m}, k_{m}^{\prime}\right)$ which yields the minimum number of samples misclassified, and take it as the optimal pair of $k$ and $k^{\prime}$. If more than one pair yield the minimum number of samples misclassified, take all these pairs as the optimal pairs of $k$ and $k^{\prime}$.

When more than one pair are obtained as optimal pairs of $k$ and $k^{\prime}$, any input sample should be classified using the $k^{\prime}-\mathrm{NN}$ rule [1-3] and $W_{1}(k)$ for all these pairs of $k$ and $k^{\prime}$. The input sample should be assigned to the majority class which is determined by the classification results obtained for all these pairs of $k$ and $k^{\prime}$. If a tie is present among classes determined by the classification results, the input sample cannot but be assigned to a class arbitrarily chosen from the classes which tie.

\section{Experimental results and discussion}

Three classification examples are presented to test the rule proposed. For the sake of comparison, the $k-\mathrm{NN}$ rule [1-3] and Wilson's edited $k-\mathrm{NN}$ rule [4] are also tested in the examples. The values of $k$ and $k^{\prime}$ are taken in the range of $1 \leqslant k \leqslant 15$ and $1 \leqslant k^{\prime} \leqslant 15$. In Wilson's edited $k-\mathrm{NN}$ rule [4], the $1-\mathrm{NN}$ rule [1-3] is usually used with the edited reference set to classify input samples, i.e., $k^{\prime}$ is restricted to 1 . In this work, however, the $k^{\prime}-\mathrm{NN}$ rule [1-3] with $k^{\prime} \geqslant 1$ is used in Wilson's edited $k-\mathrm{NN}$ rule [4].

With respect to classification accuracy, the rule proposed and Wilson's edited $k-\mathrm{NN}$ rule [4] are estimated as follows: Each sample $x$ in the data set of the example is classified using the $k^{\prime}-\mathrm{NN}$ rule [1-3] and the edited reference set, if $x$ is not in the edited reference set. If $x$ is in the edited reference set, the set formed by eliminating $x$ from the edited reference set is obtained as the reference set, and $x$ is classified using the $k^{\prime}-\mathrm{NN}$ rule [1-3] and the reference set thus obtained. The number of samples misclassified is then evaluated.

Classification accuracy of the $k-\mathrm{NN}$ rule [1-3] is estimated as follows: Each sample $x$ in the data set of the example is classified by the $k-\mathrm{NN}$ rule [1-3] in which the $k$-nearest neighbors of $x$ are obtained from the set formed by eliminating $x$ from the data set. The number of samples misclassified is then evaluated.

The first example is classification of the Iris data set [9]. The data set consists of 150 flowers which are classified into three classes, namely, Setosa, Versicolor and Virginica. Each class includes 50 flowers. Every flower is characterized by four features, and therefore, it is expressed by a four-dimensional feature vector. Table 1 shows the number of samples taken from each class to form the edited reference set in the rule proposed and that in Wilson's edited $k-\mathrm{NN}$ rule [4]. Evaluation of the number of samples misclassified has been systematically performed. The results of the rule proposed and those of Wilson's edited $k-\mathrm{NN}$ rule [4] are shown in Tables 2 and 3 , respectively. The results of the $k-\mathrm{NN}$ rule [1-3] are shown in Table 4.

The second example is classification of the butterflytype data set which was presented by Pham and Corrochano [10]. The data set consists of 51 two-dimensional points. It is classified into two classes forming the "wings" of the butterfly. Fig. 1 depicts the data set and 
Table 1

Number of samples taken from each class in editing the Iris data set

\begin{tabular}{|c|c|c|c|c|c|c|c|c|}
\hline$k$ & Setosa & Versicolor & Virginica & Total & Setosa & Versicolor & Virginica & Total \\
\hline 1 & 50 & 47 & 47 & 144 & 50 & 47 & 47 & 144 \\
\hline 2 & 50 & 47 & 45 & 142 & 50 & 47 & 47 & 144 \\
\hline 3 & 50 & 45 & 43 & 138 & 50 & 47 & 47 & 144 \\
\hline 6 & 50 & 43 & 36 & 129 & 50 & 47 & 47 & 144 \\
\hline 7 & 50 & 42 & 34 & 126 & 50 & 46 & 49 & 145 \\
\hline 8 & 50 & 40 & 34 & 124 & 50 & 47 & 48 & 145 \\
\hline 9 & 50 & 39 & 33 & 122 & 50 & 47 & 48 & 145 \\
\hline 10 & 50 & 36 & 32 & 118 & 50 & 46 & 48 & 144 \\
\hline 15 & 50 & 30 & 26 & 106 & 50 & 47 & 49 & 146 \\
\hline
\end{tabular}

Table 2

Number of samples misclassified by the rule proposed in the Iris data set

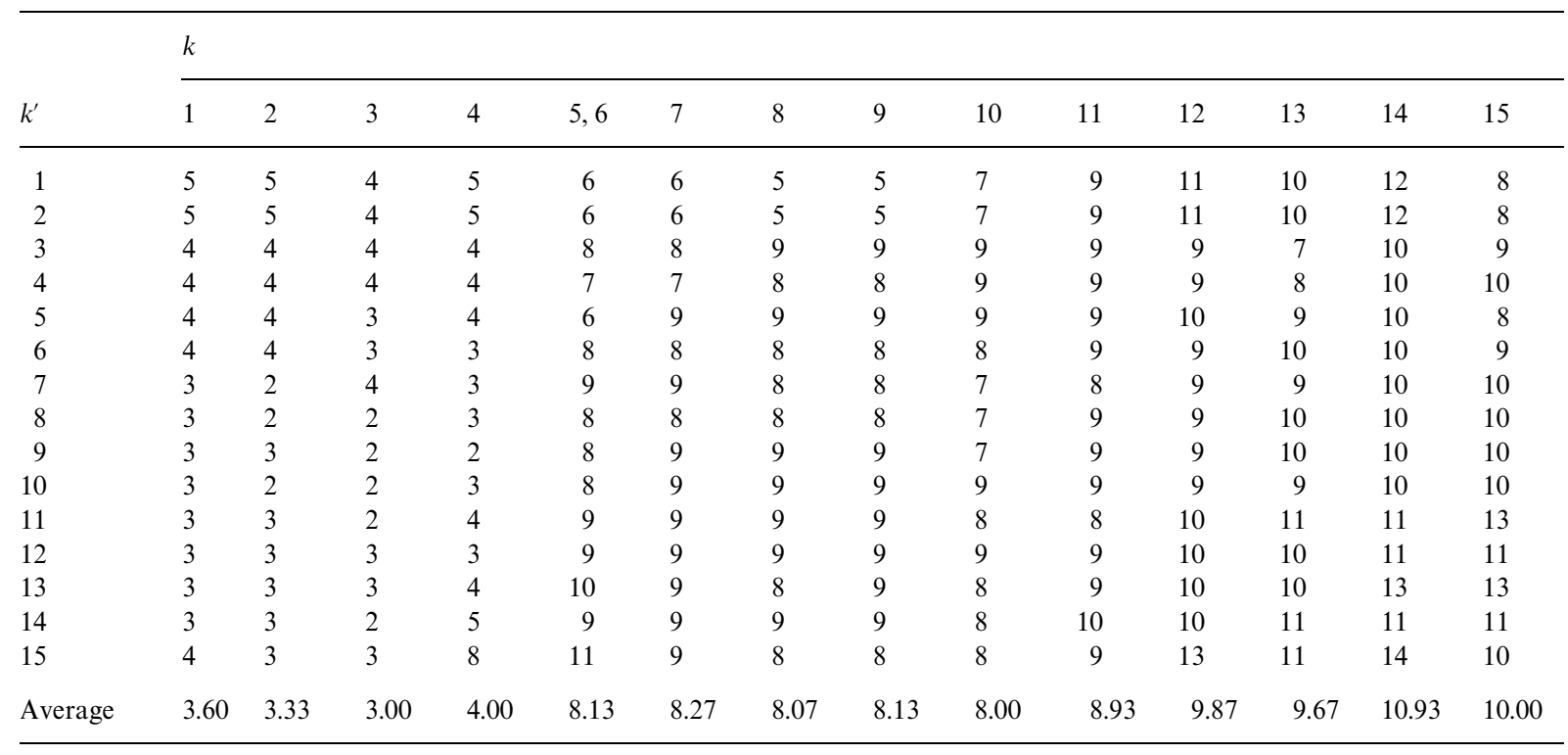

shows that there is much overlapping between two classes 1 and 2. The number of samples taken from each class to form the edited reference set is shown in Table 5. The numbers of samples misclassified are shown in Tables 6-8.

The third example is classification of the data set of 162 two-dimensional points which are shown in Fig. 2. The data set was first used by Yan [11]. As shown in Fig. 2, four classes 1-4 are present in the data set. Table 9 shows the number of samples taken from each class to form the edited reference set. Tables 10-12 show the numbers of samples misclassified.

As is clear from Tables 1, 5 and 9, the number of samples in the edited reference set in the rule proposed is equal to or less than that in Wilson's edited $k-\mathrm{NN}$ rule [4]. This is due to the fact that for any sample $x$ in the 
Table 3

Number of samples misclassified by Wilson's edited $k-\mathrm{NN}$ rule in the Iris data set

\begin{tabular}{|c|c|c|c|c|c|c|c|c|c|c|}
\hline \multirow[b]{2}{*}{$k^{\prime}$} & \multicolumn{10}{|l|}{$k$} \\
\hline & $1,2,3,4,6$ & 5 & 7,14 & 8 & 9 & 10 & 11 & 12 & 13 & 15 \\
\hline 1 & 5 & 5 & 5 & 5 & 5 & 5 & 6 & 6 & 6 & 6 \\
\hline 2 & 5 & 5 & 5 & 5 & 5 & 5 & 6 & 6 & 6 & 6 \\
\hline 3 & 4 & 4 & 4 & 4 & 4 & 4 & 5 & 5 & 5 & 5 \\
\hline 6 & 4 & 4 & 4 & 4 & 4 & 4 & 4 & 4 & 4 & 4 \\
\hline 7 & 3 & 5 & 5 & 4 & 4 & 4 & 4 & 4 & 5 & 5 \\
\hline 8 & 3 & 4 & 4 & 4 & 3 & 3 & 3 & 4 & 3 & 4 \\
\hline 9 & 3 & 3 & 4 & 3 & 5 & 4 & 5 & 3 & 3 & 4 \\
\hline 10 & 3 & 4 & 5 & 3 & 4 & 4 & 4 & 3 & 3 & 5 \\
\hline 15 & 4 & 4 & 4 & 4 & 3 & 3 & 3 & 4 & 3 & 4 \\
\hline Average & 3.60 & 4.07 & 4.33 & 3.80 & 4.13 & 4.07 & 4.47 & 4.07 & 4.07 & 4.60 \\
\hline
\end{tabular}

Table 4

Number of samples misclassified by the $k-\mathrm{NN}$ rule in the Iris data set

$k=k^{\prime}$

$\begin{array}{llllllllllllllll}1 & 2 & 3 & 4 & 5 & 6 & 7 & 8 & 9 & 10 & 11 & 12 & 13 & 14 & 15 & \text { Average }\end{array}$ $\begin{array}{llllllllllllllll}6 & 6 & 6 & 6 & 5 & 6 & 5 & 5 & 5 & 6 & 4 & 5 & 5 & 4 & 4 & 5.20\end{array}$

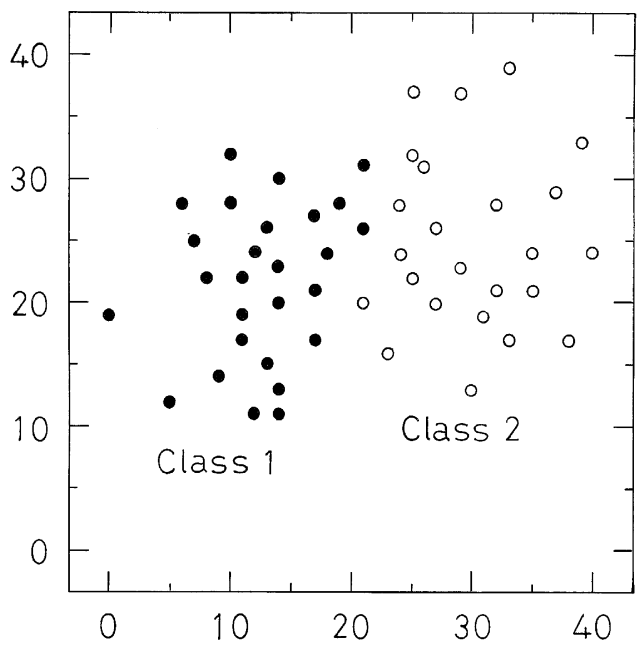

Fig. 1. Butterfly-type data set. reference set, the condition for $x$ to be included in the edited reference set in the rule proposed is severer than that in Wilson's edited $k-\mathrm{NN}$ rule [4]: In Wilson's edited $k-\mathrm{NN}$ rule [4], the majority among the $k$-nearest neighbors of $x$ must be in the class to which $x$ belongs for $x$ to be included in the edited reference set. In the rule proposed, however, all the $k$ - or $(k+\ell)$-nearest neighbors

Table 5

Number of samples taken from each class in editing the butterfly-type data set

\begin{tabular}{|c|c|c|c|c|c|c|}
\hline \multirow[b]{2}{*}{$k$} & \multicolumn{3}{|c|}{ Rule proposed } & \multicolumn{3}{|c|}{ Wilson's edited $k-\mathrm{NN}$ rule } \\
\hline & Class 1 & Class 2 & Total & Class 1 & Class 2 & Total \\
\hline 1 & 27 & 22 & 49 & 27 & 23 & 50 \\
\hline 2 & 25 & 20 & 45 & 27 & 23 & 50 \\
\hline 3 & 25 & 18 & 43 & 25 & 24 & 49 \\
\hline 4 & 24 & 17 & 41 & 26 & 24 & 50 \\
\hline 5 & 22 & 17 & 39 & 26 & 24 & 50 \\
\hline 6 & 21 & 15 & 36 & 26 & 24 & 50 \\
\hline 7 & 21 & 14 & 35 & 27 & 24 & 51 \\
\hline 8 & 21 & 14 & 35 & 26 & 24 & 50 \\
\hline 9 & 21 & 14 & 35 & 25 & 23 & 48 \\
\hline 10 & 19 & 13 & 32 & 26 & 23 & 49 \\
\hline 11 & 18 & 13 & 31 & 25 & 23 & 48 \\
\hline 12 & 17 & 11 & 28 & 25 & 23 & 48 \\
\hline 13 & 16 & 11 & 27 & 25 & 23 & 48 \\
\hline 14 & 16 & 10 & 26 & 26 & 23 & 49 \\
\hline 15 & 16 & 10 & 26 & 26 & 23 & 49 \\
\hline
\end{tabular}


Table 6

Number of samples misclassified by the rule proposed in the butterfly-type data set

\begin{tabular}{|c|c|c|c|c|c|c|c|c|c|c|c|c|}
\hline \multirow[b]{2}{*}{$k^{\prime}$} & \multicolumn{12}{|l|}{$k$} \\
\hline & 1 & 2 & 3,4 & 5 & 6 & $7,8,9$ & 10 & 11 & 12 & 13 & 14 & 15 \\
\hline 1 & 1 & 1 & 1 & 0 & 0 & 0 & 0 & 0 & 1 & 1 & 1 & 1 \\
\hline 2 & 1 & 1 & 1 & 0 & 0 & 0 & 0 & 0 & 1 & 1 & 1 & 1 \\
\hline 3 & 1 & 0 & 1 & 0 & 1 & 1 & 1 & 1 & 1 & 1 & 1 & 1 \\
\hline 6 & 0 & 1 & 1 & 1 & 1 & 1 & 1 & 1 & 1 & 1 & 1 & 1 \\
\hline 7 & 0 & 1 & 1 & 1 & 1 & 1 & 1 & 1 & 1 & 1 & 2 & 1 \\
\hline 8 & 0 & 1 & 1 & 0 & 1 & 1 & 1 & 1 & 1 & 1 & 1 & 1 \\
\hline 9 & 1 & 1 & 1 & 1 & 1 & 1 & 1 & 1 & 2 & 1 & 2 & 2 \\
\hline 10 & 1 & 1 & 1 & 1 & 1 & 1 & 1 & 1 & 1 & 1 & 2 & 2 \\
\hline 15 & 1 & 1 & 1 & 1 & 2 & 2 & 2 & 2 & 2 & 2 & 3 & 3 \\
\hline Average & 0.80 & 0.87 & 1.00 & 0.67 & 0.93 & 1.07 & 0.93 & 0.87 & 1.53 & 1.47 & 1.73 & 1.60 \\
\hline
\end{tabular}

of $x$ obtained from the procedure E- 1 must be in the class to which $x$ belongs.

In the rule proposed, the condition for a sample to be included in the edited reference set becomes severer with

Table 7

Number of samples misclassified by Wilson's edited $k-\mathrm{NN}$ rule in the butterfly-type data set
Table 8

Number of samples misclassified by the $k-\mathrm{NN}$ rule in the butterfly-type data set

\begin{tabular}{rrrrrrrrrrrrrrrl}
\hline$k=k^{\prime}$ & \multicolumn{11}{c|}{} & & \\
\hline 1 & 2 & 3 & 4 & 5 & 6 & 7 & 8 & 9 & 10 & 11 & 12 & 13 & 14 & 15 & Average \\
1 & 1 & 2 & 1 & 1 & 1 & 0 & 1 & 3 & 2 & 3 & 3 & 3 & 2 & 2 & 1.73
\end{tabular}

\begin{tabular}{|c|c|c|c|c|c|c|}
\hline \multirow[b]{2}{*}{$k^{\prime}$} & \multicolumn{6}{|l|}{$k$} \\
\hline & 1,2 & 3 & $\begin{array}{l}4,5 \\
6,8\end{array}$ & 7 & $\begin{array}{l}9,11, \\
12,13\end{array}$ & $\begin{array}{l}10,14, \\
15\end{array}$ \\
\hline 1 & 1 & 1 & 1 & 1 & 1 & 1 \\
\hline 2 & 1 & 1 & 1 & 1 & 1 & 1 \\
\hline 3 & 2 & 2 & 2 & 2 & 2 & 2 \\
\hline 4 & 1 & 1 & 1 & 1 & 1 & 1 \\
\hline 5 & 1 & 1 & 1 & 1 & 1 & 1 \\
\hline 6 & 1 & 1 & 1 & 1 & 1 & 1 \\
\hline 7 & 0 & 2 & 1 & 0 & 2 & 1 \\
\hline 8 & 1 & 2 & 2 & 1 & 1 & 1 \\
\hline 9 & 2 & 3 & 3 & 3 & 3 & 3 \\
\hline 10 & 2 & 3 & 3 & 2 & 3 & 3 \\
\hline 11 & 3 & 3 & 3 & 3 & 3 & 3 \\
\hline 12 & 2 & 3 & 3 & 3 & 2 & 2 \\
\hline 13 & 2 & 3 & 3 & 3 & 2 & 2 \\
\hline 14 & 2 & 3 & 3 & 2 & 2 & 2 \\
\hline 15 & 2 & 3 & 3 & 2 & 3 & 3 \\
\hline Average & 1.53 & 2.13 & 2.07 & 1.73 & 1.87 & 1.80 \\
\hline
\end{tabular}

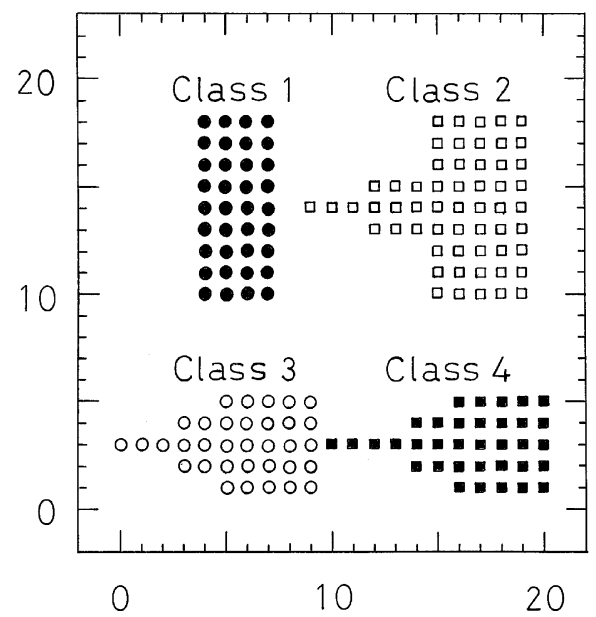

Fig. 2. Data set of 162 points. 
Table 9

Number of samples taken from each class in editing the data set of 162 points

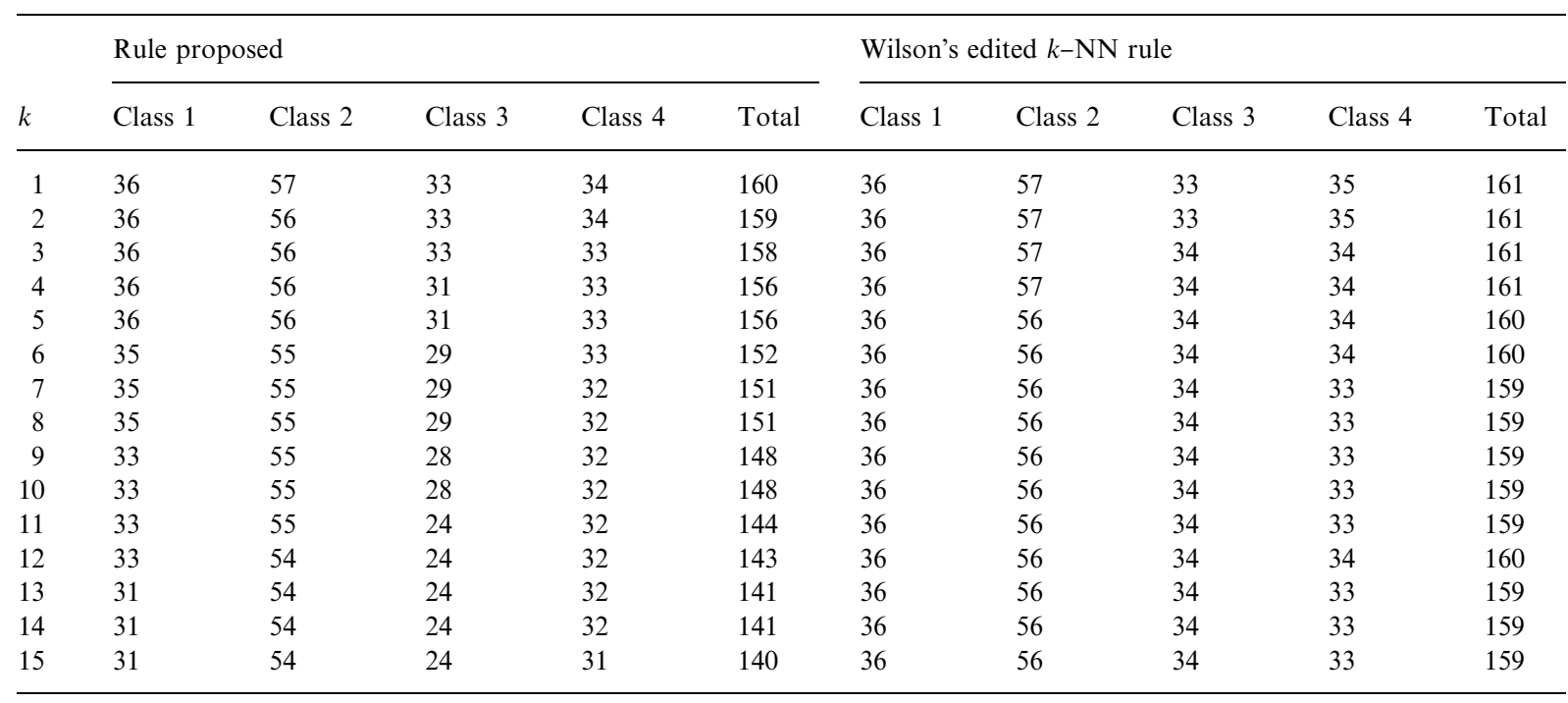

Table 10

Number of samples misclassified by the rule proposed in the data set of 162 points

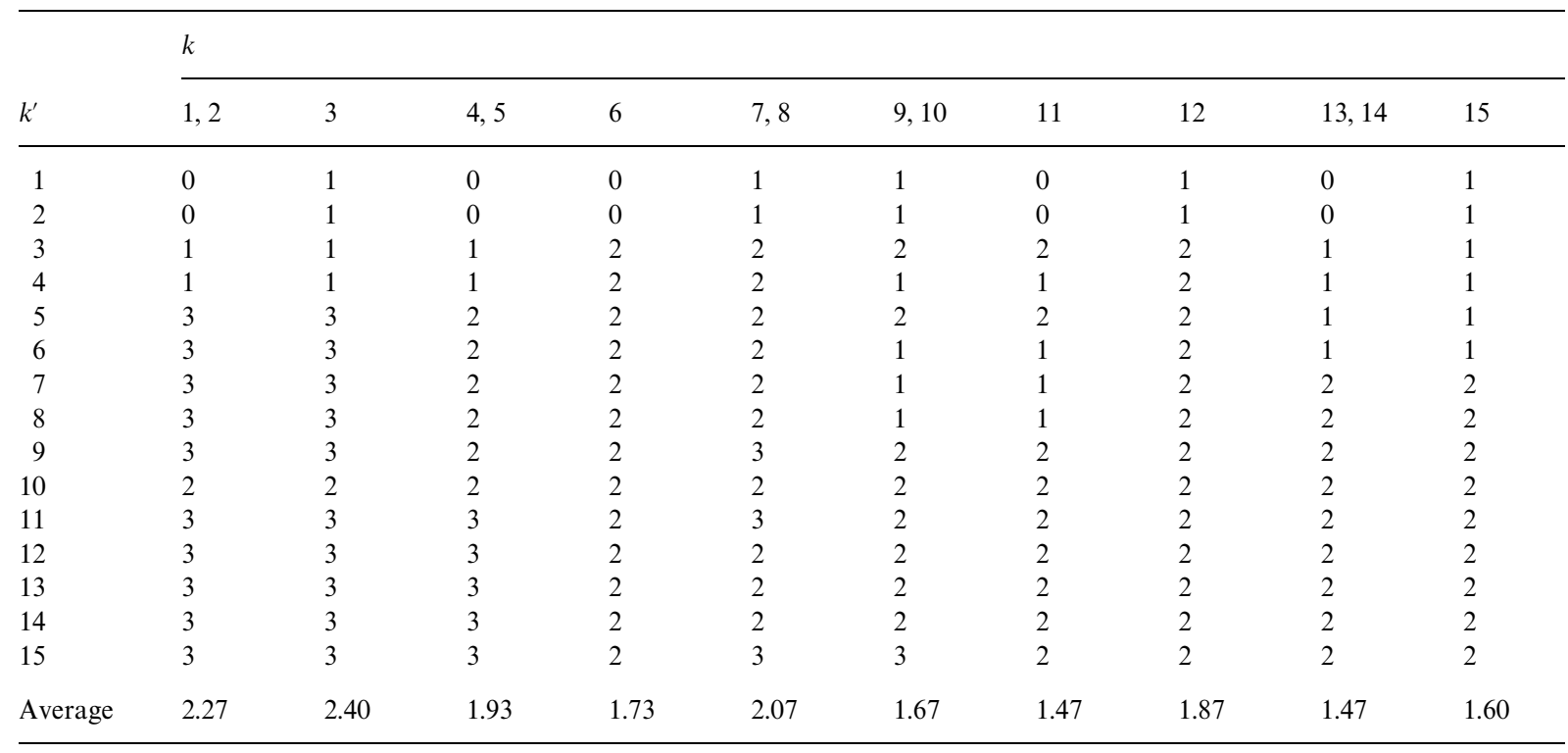

increasing $k$. Therefore, the number of samples in the edited reference set becomes smaller with increasing $k$. This tendency is found in Tables 1, 5 and 9. In the tables, however, the number of samples in the edited reference set in Wilson's edited $k-\mathrm{NN}$ rule [4] is not so changed by $k$. In the special case of $k=1$ and $\ell=0$, the edited reference set in the rule proposed is identical with that in Wilson's edited $k-\mathrm{NN}$ rule [4]. This special case is found for $k=1$ in Table 1 .
From Tables 2-4, the minimum number of samples misclassified in the Iris data set [9] is estimated to be 2 in the rule proposed, 3 in Wilson's edited $k-\mathrm{NN}$ rule [4] and 4 in the $k-\mathrm{NN}$ rule [1-3]. Further, it is found from Tables 2-4 that the results of the rule proposed for $k=2$ and 3 are much better than those of the $k-\mathrm{NN}$ rule [1-3] and Wilson's edited $k-\mathrm{NN}$ rule [4] for $1 \leqslant k \leqslant 15$ : The average number of samples misclassified in the range of $1 \leqslant k^{\prime} \leqslant 15$ is very small for $k=2$ and 3 in the rule 
Table 11

Number of samples misclassified by Wilson's edited $k-\mathrm{NN}$ rule in the data set of 162 points

\begin{tabular}{rlll}
\hline & $k$ & & \\
\cline { 2 - 3 }$k^{\prime}$ & 1,2 & $3,4,5,6,12$ & $7,8,9,10,11$, \\
& & & $13,14,15$ \\
\hline 1 & 1 & 0 & 1 \\
2 & 1 & 0 & 1 \\
3 & 1 & 1 & 1 \\
4 & 1 & 1 & 1 \\
5 & 2 & 3 & 3 \\
6 & 2 & 3 & 3 \\
7 & 3 & 3 & 3 \\
8 & 2 & 3 & 3 \\
9 & 3 & 3 & 3 \\
10 & 2 & 3 & 3 \\
11 & 2 & 3 & 3 \\
12 & 2 & 3 & 3 \\
13 & 3 & 3 & 3 \\
14 & 3 & 3 & 3 \\
15 & 3 & 3 & 2.47 \\
Average & 2.07 & 2.33 & \\
\hline
\end{tabular}

Table 12

Number of samples misclassified by the $k-\mathrm{NN}$ rule in the data set of 162 points

$k=k^{\prime}$

$\begin{array}{llllllllllllllll}1 & 2 & 3 & 4 & 5 & 6 & 7 & 8 & 9 & 10 & 11 & 12 & 13 & 14 & 15 & \text { Average }\end{array}$

$\begin{array}{llllllllllllllll}1 & 1 & 1 & 1 & 2 & 2 & 3 & 3 & 3 & 3 & 3 & 2 & 3 & 3 & 3 & 2.27\end{array}$

proposed. However, Table 2 shows that the results of the rule proposed for $k \geqslant 5$ are not good. This will be due to serious decrease of samples in the edited reference set for $k \geqslant 5$.

From Tables 6-8, the minimum number of samples misclassified in the butterfly-type data set [10] is obtained as zero in the rule proposed, Wilson's edited $k-\mathrm{NN}$ rule [4] and the $k-\mathrm{NN}$ rule [1-3]. Tables $6-8$ show that in the evaluation of the average number of samples misclassified in the range of $1 \leqslant k^{\prime} \leqslant 15$, the results of the rule proposed for $1 \leqslant k \leqslant 11$ are much better than those of the $k-\mathrm{NN}$ rule [1-3] and Wilson's edited $k-\mathrm{NN}$ rule [4] for $1 \leqslant k \leqslant 15$.

From Tables 10-12, the minimum number of samples misclassified in the data set of 162 points [11] is evaluated as zero in the rule proposed and Wilson's edited $k-\mathrm{NN}$ rule [4], and as 1 in the $k-\mathrm{NN}$ rule [1-3]. Tables 10-12 show that in the evaluation of the average number of samples misclassified in the range of $1 \leqslant k^{\prime} \leqslant 15$, the results of the rule proposed for $9 \leqslant k \leqslant 15$ are much better than those of the $k-\mathrm{NN}$ rule [1-3] and Wilson's edited $k-\mathrm{NN}$ rule [4] for $1 \leqslant k \leqslant 15$.

\section{Conclusions}

A new edited $k-\mathrm{NN}$ rule has been proposed, and its performance is investigated using three classification examples. In the examples, the rule proposed has yielded better results than the $k-\mathrm{NN}$ rule [1-3] and Wilson's edited $k-\mathrm{NN}$ rule [4]. This results from the fact that good edited reference sets are obtained in the rule proposed. The condition for a sample to be included in the edited reference set in the rule proposed is severer than that in Wilson's edited $k-\mathrm{NN}$ rule [4]. In the rule proposed, every sample $y$ in the edited reference set for any $k$ must be surrounded by its $k$ - or $(k+\ell)$-nearest neighbors which are all in the class to which $y$ belongs. Accordingly, every sample $y$ in the edited reference set is very proper as a typical sample in the class to which $y$ belongs.

The number of samples in the edited reference set in the rule proposed is shown to be equal to or less than that in Wilson's edited $k-\mathrm{NN}$ rule [4], and it decreases with increasing $k$. Therefore, editing the reference set using a too large value of $k$ is not good, and $k$ should be properly determined in some range of $k$. If the values of $k$ and $k^{\prime}$ are optimally determined by taking the procedures from $k-1$ to $k-3$, the rule proposed will yield good results in many pattern classification problems.

\section{Summary}

A new edited $k-\mathrm{NN}$ rule has been proposed. In the rule, the condition for a sample $x$ to be included in the edited reference set is that all the $k$ - or $(k+\ell)$-nearest neighbors of $x$ must be in the class to which $x$ belongs. Here $\ell$ denotes the number of samples which tie with the $k$ th nearest neighbor of $x$ with respect to the distance from $x$. Accordingly, this condition is severer than that in Wilson's edited $k-\mathrm{NN}$ rule, and every sample in the reference set in the rule proposed is very proper as a typical sample in the class to which it belongs. The number of samples in the edited reference set in the rule proposed is equal to or less than that in Wilson's edited $k-\mathrm{NN}$ rule, and it decreases with increasing $k$.

Three classification examples are presented to test the rule proposed. The $k-\mathrm{NN}$ rule and Wilson's edited $k-\mathrm{NN}$ rule are also performed in the examples. The rule proposed is found to yield better results than the $k-\mathrm{NN}$ rule and Wilson's edited $k-\mathrm{NN}$ rule. Any input sample can be classified using the $k^{\prime}-\mathrm{NN}$ rule and the edited reference set. If the values of $k$ and $k^{\prime}$ are optimally determined by taking the procedures presented in this work, the rule proposed will yield good results in many pattern classification problems. 


\section{References}

[1] E. Fix, J.L. Hodge Jr., Discriminatory analysis, nonparametric discrimination, consistency properties, U.S. Air Force Sch. Aviation Medicine, Randolf Field, Texas, Project 21-49-004, Contract AF 41 (128)-31, Rep. 4, 1951.

[2] R.O. Duda, P.E. Hart, Pattern Classification and Scene Analysis, Wiley, New York, 1973.

[3] P.A. Devijver, J. Kittler, Pattern Recognition: A Statistical Approach, Prentice-Hall, London, 1982.

[4] D.L. Wilson, Asymptotic properties of nearest neighbor rules using edited data, IEEE Trans. System Man Cybernet. 2 (1972) 408-421.

[5] L.I. Kuncheva, Fitness functions in editing $k-\mathrm{NN}$ reference set by genetic algorithms, Pattern Recognition 30 (1997) 1041-1049.
[6] P.A. Devijver, J. Kittler, On the edited nearest neighbor rule, Proc. 5th Int. Conf. on Pattern Recognition, 1980, pp. 72-80.

[7] B.V. Dasarathy, Minimal consistent set (MCS) identification for optimal nearest neighbor decision systems design, IEEE Trans. System Man Cybernet. 24 (1994) 511-517.

[8] D. Goldberg, Genetic Algorithms in Search, Optimization and Machine Learning, Addison-Wesley, Reading, MA, 1989.

[9] E. Anderson, The irises of the Gaspe peninsula, Bull. Am. Iris Soc. 59 (1935) 2-5.

[10] D.T. Pham, E.J.B. Corrochano, Self-organizing neuralnetwork-based pattern clustering method with fuzzy outputs, Pattern Recognition 27 (1994) 1103-1110.

[11] H. Yan, Prototype optimization for nearest neighbor classifiers using a two-layer perceptron, Pattern Recognition 26 (1993) 317-324.

\begin{abstract}
About the Author-KAZUO HATTORI received his Ph.D. degree in Applied Physics from Tokyo University, Japan, in 1977. He is at present an Associate Professor in the Department of Electrical Engineering and Electronics at Toyohashi University of Technology. His research interests are in the areas of cluster analysis, fuzzy inference and decision theory.
\end{abstract}

About the Author-MASAHITO TAKAHASHI received his M.E. degree in Electrical Engineering and Electronics from Toyohashi University of Technology, Japan, in 1997. He is currently engaged in Ph.D. research in the Department of Electrical Engineering and Electronics, Toyohashi University of Technology. 